\title{
Evaluating Professional Search: A German Construction Law Use Case
}

\author{
Wei Li \\ ADAPT, School of Computing, Dublin City University \\ Dublin, Ireland \\ wei.li@adaptcentre.ie
}

\begin{abstract}
We present a real world case study for the evaluation of professional search focusing on German construction law. Reliable identification of relevant previous cases is an important part of many legal disputes, and currently relies on domain expertise acquired over a lengthy professional career. We describe our experiences from the development of a Cranfield type test collection for a German construction law dataset to enable research into the development of search technologies for new tools which are less dependent on expert knowledge. We describe examination of the search needs of lawyers, the development of a set of search queries created by lawyers, and our experiences in collecting expert relevance data for the completion of a test collection for legal search. Important findings of this latter process are the need for individuals with expert legal training to assess relevance, and the identification of context dependence in determining relevance. While the cost of the development of this test collection was found to be very high, we demonstrate its value in terms of identifying the effectiveness of legal search methods and in identifying research directions for legal case search.
\end{abstract}

\section{CCS CONCEPTS}

- Information systems $\rightarrow$ Relevance assessment; Expert search.

\section{KEYWORDS}

Professional Search, Legal Search, Evaluation, Expert Relevance Assessment

\section{ACM Reference Format:}

Wei Li and Gareth J. F. Jones. 2020. Evaluating Professional Search: A German Construction Law Use Case. In Forum for Information Retrieval Evaluation (FIRE '20), December 16-20, 2020, Hyderabad, India. ACM, New York, NY, USA, 5 pages. https://doi.org/10.1145/3441501.3441677

\section{INTRODUCTION}

Evaluation of search effectiveness for non-expert search tasks typically adopts the use of test collections using the Cranfield evaluation paradigm. For non-expert search tasks search topics can be created by members of the general public in the form of statements of information need on widely known issues. Corresponding relevance

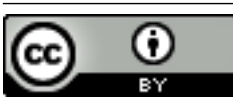

This work is licensed under a Creative Commons Attribution International 4.0 License. FIRE '20, December 16-20, 2020, Hyderabad, India

(C) 2020 Copyright held by the owner/author(s).

ACM ISBN 978-1-4503-8978-5/20/12.

https://doi.org/10.1145/3441501.3441677

\author{
Gareth J. F. Jones \\ ADAPT, School of Computing, Dublin City University \\ Dublin, Ireland \\ gareth.jones@adaptcentre.ie
}

assessments can then be made reliably by similarly non-expert judges. A subject of much less attention within the information retrieval (IR) research community is the evaluation of professional search, as undertaken by task experts as part of their work. Such users often have very specific, much more specialised information needs than those generally considered within IR research. However, it is generally implicitly assumed that methods found to be most effective for general search, will also be the best to meet user information needs in very different professional settings.

In this paper, we examine the development and testing of a professional search test collection for legal search, representing evaluation of the search activities of specialist lawyers. In this setting, it is only possible to obtain representative search queries from lawyers themselves, and we find that corresponding relevance judgements can similarly only be provided by suitably qualified lawyers. The complexity of information needs and necessary involvement of lawyers in the development of a test collection, means that, as we demonstrate, the cost of test collection development is extremely high in terms of both time and money. It is thus crucial to select items for relevance assessment extremely carefully with particular consideration of the potential relevance or usefulness of retrieved items to a lawyer. In this work, we propose a method which utilizes domain-specific information to generate query variants for creation of an assessment pool.

The remainder of this paper is structured as follows: Section 2 overviews related work; Section 3 introduces our legal document set, sample query development, and our relevance assessment strategy; Section 4 explains our indexing of a large legal document collection; Section 5 describes our relevance assessment process, with analysis of the relevance assessments obtained; Section 6 introduces an IR experiment which demonstrates the potential real-world value of our professional test collection; and finally, future work is suggested.

\section{RELATED WORK}

There is very limited existing work examining evaluation of professional search. There are though, some notable examples in area of legal IR. Maxwell and Schafer [4] describe a method of knowledge engineering and natural language processing methods for legal search. Saias and Quaresma [6] propose a method to create an ontology to enrich web legal documents to improve legal IR. In [8], Noortwijk proposed an integrated legal IR method which combines a large legal resource for educational purposes. Discussions and analysis are presented in various research papers, but no significant experiments and results are clearly presented. In our work, we address a real use case problem which makes our experiment and results more reliable for application in professional settings than existing work. 
In [3] [9], the authors discuss and define the dimension of 'relevance' from six aspects according to the characteristic of legal data. This is similar to our goal for which we define a new criteria of relevance. The key difference is that instead of building a relevance dimension based on the relation between query and documents, our relevance criteria is defined based on feedback from real world lawyers, and addresses concepts focusing on the query side. Moffat and Scholer [5] show that the effectiveness of employing query variation in document pooling is as strong as that of using retrieval system variation. We also employ a pooling method based on query variants, our work is different from that of Moffat and Scholer's since their query variants are built by crowd workers, whereas we use the annotations provided with the queries to create query variants. Also the pooling methods we employ to carry out relevance assessment are different from existing research, such as [2] and [7].

\section{DATA AND PROCESSING}

In this section we describe the document collection and the query creation process used for our study.

\subsection{Document Collection}

A real world legal case document collection was provided to us by a large legal technology company. This document collection focuses on German Construction Law, and contains approx. 1,000,000 court German language decision documents in XML format. Each document pertains to the court decision for one case. The time frame of these legal documents covers the period 1950s to 2017. Together with the corpus, the legal company also provided a related hierarchical taxonomy which includes 4,636 legal concepts which can be assigned to the cases.

\subsection{Query Creation}

Test queries were extracted from summary notes made relating to current cases by lawyers. These notes typically describe information needs expressed using professional descriptions. 31 sample query statements were extracted from these notes by lawyers as expression of information needs likely to be encountered by a lawyer when they are working on a case using these notes. The queries vary in length from from 36 to 421 words and covered 7 sub-domain as shown in Table 1. Within 31 sample queries, 27 queries' length are in range 100 to 200 words, 3 are less than 100 words, and 1 query has 421 words length. The 7 sub-domains and query distributions are shown in Table 1 .

Legal information needs are more complex than those in more general areas, such as web search, this complexity is further increased for our dataset by the use of German language. To assist our understanding of the queries, lawyers provided additional information accompanied with each sample query. This information included: keywords, 10 manually located relevant documents from the document collection, relevant concepts taken from the supplied hierarchical legal taxonomy, and links to relevant legislation.

Further details of the accompanying annotations are as follows:

Keywords: The lawyers manually extracted keywords from each query statement and highlighted phrases relating to these keywords based on their legal knowledge. An example of query statement
Table 1: Sub-domains and the number of sample queries

\begin{tabular}{lc}
\hline Sub-domain & $\begin{array}{c}\text { Number of Queries } \\
\text { on this sub-domain }\end{array}$ \\
\hline Mängel (defects) & 17 \\
Vertrag (contract) & 8 \\
Nachtrag (appendix contract) & 2 \\
Zeit (time) & 1 \\
Architektenrecht (Architectural law) & 1 \\
Forderung (demand) & 1 \\
Leistungsänderung (Power change) & 1 \\
\hline
\end{tabular}

Query Statement: Der Mandant (Unternehmer Gewerk Gartenund Landschaftsbau) hat eine offene Restwerklohnforderung gegen seinen Auftraggeber (Generalunternehmer) geltend gemacht. Neben dem restlichen Werklohn möchte er hierfür auch eine Bauhandwerkersicherheit nach $§ 648$ a a.F. BGB einklagen. Da über die Werklohnforderung noch eine umfangreiche Beweisaufnahme erforderlich sein wird, möchte er die Bauhandwerkersicherheit möglichst schon vorab erhalten, um seinen Werklohnanspruch abzusichern.

Keywords: Werklohnklage Bauhandwerkersicherung Teilurteil

Figure 1: Example Query with Keywords highlighted.

textit $<$ registereintrag class $=$ "core-concept-annotation annotation" $t$ tle $=$ Nachtrag" type $=$ "core-concept" id="ui-id-134">Hierdurch entstehen <registereintrag class="annotation" title="Besondere Vergütung für vertraglich nicht vorgesehene Leistung (Preisermittlung bei fehlender Vergütungsvereinbarung,Vergütung für eigenmächtige Leistungen)" id="13293" type="concept"> erhebliche Zusatzkosten </registereintrag $>$ die die Mandantin im Rahmen eines Nachtragsangebots geltend macht.

\section{Figure 2: Core concept annotations of a query.}

with highlighted phrases and extracted keywords are shown is shown in Figure 1.

Annotations: The lawyers manually labelled the queries with concepts extracted from the provided legal taxonomy.

They labelled each query with various legal aspects it addressed, In order to fully interpret the query, in addition to concept annotation, they also annotated each query with summarized phrases based on their domain knowledge, we refer these phrases as 'core concepts'. In total, 88 core concept annotations were assigned to the 31 sample queries. An example of core concept annotations is shown in Figure 2.

Concept tagger usually show the full path of the taxonomy from the root concept to the leaf one.

\section{DOCUMENT INDEXING}

The legal documents are long and complex with manual concept annotations. It is not clear what the best search unit is or how they should be indexed. To begin our investigation of this topic, we built three alternative indexes. 
Table 2: Query variants generation

\begin{tabular}{ll}
\hline Variants & Query Variant Components \\
\hline Query V1 & Original query statement (OQS) \\
Query V2 & Keywords only \\
Query V3 & OQS + keywords \\
Query V4 & keywords + synonyms \\
Query V5 & OQS + labelled concepts \\
Query V6 & OQS + labelled core concepts \\
Query V7 & OQS + labelled concepts + labelled core concepts \\
Query V8 & Keywords + labelled concepts + labelled core concepts \\
\hline
\end{tabular}

Word-level index: A word-level index for each full document was created using Solr ${ }^{1}$. The internal BM25 retrieval model with standard parameter settings was used to perform search. For each query, Solr returned a ranked retrieval list. Eight ranked output retrieval lists were obtained for the variants of each query statement. The top $N$ ranked documents were taken from each list for inclusion in the relevance assessment pool.

Concept index: Each document contains a meta tag containing associated concepts taken from the provided legal taxonomy. We used Solr to build a separate concept index for each document using only these concept labels.

Fragment index: Since the documents are long, we segmented each one into coherent fragments and built a further index treating them as independent search units again using Solr. Fragments were extracted from the document based on their assigned XML tags, and lexical segmentation of long text sub-sections using the C99 segmentation algorithm [1]. The processed segments include the following attributes:

- docid: the id of the document this fragment came from

- section: what section this fragment belongs to

- type: type of the segment

- fragment_text: the content of the fragment

\section{RELEVANCE ASSESSMENT}

Having created the queries and indexed the documents, the final stage in the development of our test collection for investigation of professional legal search, was to gather relevance information for the documents.

Pooling: The standard method to do this is to created pool subset of documents for each query by merging the search output of a number of different search tools when searching using this query. Rather than adopt this standard approach, in this investigation, we employed a method of generating query variants for application to a single search system. The top ranked elements of the retrieval lists were then combined to form the assessment pool based on their frequency of appearance in query variants' result rankings.

To create the query variant, we used the concepts, core concepts, synonyms and tagging information provided with this each query to construct diverse alternative forms of the queries. The very different

\footnotetext{
${ }^{1}$ https://lucene.apache.org/solr/
}

contents of the query variants helped to identify a wide variety of potentially relevant documents. Overall, eight query variants were developed for each sample query are shown in Table 2.

For each sample query the top 15 ranked documents retrieved using the alternative query statements and the word-level index were included in the initial pool. After removing duplicates, approximately 80 to 100 documents were left in each pool. It was clear from analysis of topic statements and documents by a native German speakers, that relevance assessment must be conducted by legal experts. An important finding was that, on average, it took an experienced legal expert 10 minutes to assess one document. The limited availability of the lawyers due to professional commitments, meant that the assessment process became very lengthy. The extended duration of the assessment process meant that eventually only 26 queries could be completed within the timeframe of our investigation. These queries cover all 7 sub-topics as follows: Mängel (14), Vertrag (7), Nachtrag (1), Zeit (1), Architektenrecht (1), Forderung (1), Leistungsänderung (1).

Assessment Scale: In order to collect relevance data for each query, suitable relevance criteria had to be determined. A relevance scale rating is widely used in IR relevance judgement, either using graded relevance criteria or scale of 1 to 10 . After discussion with the legal experts we agreed the following assessment scale:

- It is what I am looking for:

Relevant document, related to query, lawyers will read

- On topic, relevant but will not read:

Relevant document, related to query, but lawyer may not read. Typically because this document is a well known case for lawyers.

- On topic, but not relevant:

Document is on the same general topic with sample query but not relevant to query case. Legal experts will not read.

- Not relevant at all :

Documents totally not relevant to query.

Assessment Analysis: For an initial trial assessment, we used two queries (query 29 and 31) to examine the reasoning among two different lawyers. Their ratings showed large relevancy disagreements. For example, for query 29, where the assessment pool contained 93 documents, 69 different relevancy levels were assigned by the two assessors, with 37 of them being totally opposite.

The two legal assessors were interviewed to discuss these disagreements. The reason was revealed to be that they focused on different sub-topics of query content. The discussion showed us that lawyers can have distinct interpretations of the same detailed query statement. The lawyers claimed that they were not surprised that they have given ratings for the same document, since the same query could be entered by different lawyers an alternative intention. We decided to assign each query assessment pool to two legal experts for assessing, and for a particular document, if it was rated as relevant by either assessor, we consider it relevant.

Eight legal experts contributed to relevance judgement task. Each of them was assigned 5 or 6 queries for assessment.

Finally, 26 queries were assessed by the 8 legal experts. On average, around $25 \%$ documents in pool for each query were found to be relevant. Legal experts gave positive feedback for the contents 
Table 3: Evaluation Results for different indexing methods

\begin{tabular}{lcccccccc}
\hline Methods & Num_q & Num_rel & Num_ret & MAP & P@5 & P@10 & P@15 & P@20 \\
\hline Baseline & 26 & 975 & 261 & 0.0255 & 0.1462 & 0.1000 & 0.0769 & 0.0654 \\
Baseline+CoreConcept & 26 & 975 & 300 & 0.0482 & 0.2080 & 0.1680 & 0.1440 & 0.1280 \\
ConIndex & 26 & 975 & 28 & 0.0013 & 0.0077 & 0.0038 & 0.0051 & 0.0058 \\
FragIndex_SUM & 26 & 975 & $\mathbf{3 9 4}$ & $\mathbf{0 . 0 6 5 7}$ & $\mathbf{0 . 2 4 6 2}$ & $\mathbf{0 . 1 8 0 8}$ & $\mathbf{0 . 1 5 6 4}$ & $\mathbf{0 . 1 4 4 2}$ \\
FragIndex_MEAN & 26 & 975 & 394 & 0.0302 & 0.1000 & 0.0731 & 0.0641 & 0.0538 \\
FragIndex_NoLower & 26 & 975 & 394 & 0.0542 & 0.2077 & 0.1538 & 0.1256 & 0.1231 \\
Baseline+FragIndex_SUM & 26 & 975 & 261 & 0.0530 & 0.2385 & $\mathbf{0 . 1 9 2 3}$ & 0.1564 & 0.1362 \\
Baseline+FragIndex_MEAN & 26 & 975 & 261 & 0.0465 & 0.2000 & 0.1731 & 0.1462 & 0.1173 \\
Baseline+FragIndex_NoLower & 26 & 975 & 261 & 0.0549 & 0.2769 & 0.1962 & 0.1641 & 0.1288 \\
\hline
\end{tabular}

of these pools where they considered most documents to be either relevant or topic related. As mentioned, for each sample query, 10 relevant documents were supplied with the query. We combined these with collected relevance judgements together to form our test collection for evaluation.

\section{EXPERIMENTAL INVSTIGATION}

We conducted a series of experiments to examine the utility of our test collection. Due to space limitations, we present only the results for using the 3 indexes with the original query statements for search, and select only one run to examine the core concepts importance assigned to the queries by lawyers, by adding them to query statement on word level indexing (Baseline+CoreConcept) The standard BM25 retrieval model was used as the baseline method (Baseline) to compare with concept indexing (ConIndex) and fragment index (FragIndex). For the concept index, its query was the concepts assigned to the query by the lawyers. For fragment indexing, since fragment indexing only ranked fragments, we examined three methods to process the retrieved fragment result list to form a document list for evaluation. If multiple fragments from the same document occur on the retrieval list:

- Sum of their score used as document score (FragIndex_SUM)

- Mean of their scores used as document score (FragIndex_MEAN)

- Ignore lower ranked fragments, use top ranked fragment score as document score (FragIndex_NoLower)

These three fragment results combined with Solr retrieval list were also examined separately.

The 26 assessed queries are used for our experiments. The top 1000 documents for each retrieval list were evaluated using the standard NIST trec_eval tool. Discussion with lawyers indicated that they were conducting precision oriented searches, so we report Precision@cut off rank and mean average precision (MAP) to examine the effectiveness of our alternative indexing methods. The results in Table 3 indicate that addition of core concepts is an effective means to improve retrieval results, with MAP (run "Baseline+CoreConcept") improving by $89 \%$ relative to the baseline. Runs using the original query statement to search the fragment index outperforms the other two indexes, and retrieve 133 more relevant documents than for the Baseline. FragIndex_SUM run achieved the best overall retrieval results. The concept index performs poorly compared to other runs, we believe that this is caused by sparsity concept annotations in the documents and the queries.

\section{CONCLUSIONS AND FUTURE WORK}

In this paper we presented a case study examining the evaluation of professional search for an expert legal search task. A relevance rating scale was defined for this task based on criteria agreed with legal professionals. While expensive and time consuming to construct, this expert test collection immediately provided valuable feedback and insights into the behaviour of our approach to legal IR. These clearly indicate strengths and weaknesses, and suggest directions for further research. These insights would not be available from user feedback, which was previously our only means of determining search effectiveness in this task domain. How to make the test collection construction process more affordable for professional search tasks such as this is an important challenge for further work.

\section{ACKNOWLEDGMENTS}

The ADAPT Centre for Digital Content Technology (www.adaptcentre. ie) at Dublin City University is funded by the Science Foundation Ireland Research Centres Program (Grant 13/RC/2106) and is cofunded by the European Regional Development Fund.

\section{REFERENCES}

[1] Freddy Y. Y. Choi. 2000. Advances in Domain Independent Linear Text Segmentation. In Proceedings of the 1st North American Chapter of the Association for Computational Linguistics Conference (Seattle, Washington) (NAACL 2000). Association for Computational Linguistics, USA, 26-33.

[2] Cyril Cleverdon. 1997. The Cranfield Tests on Index Language Devices. Morgan Kaufmann Publishers Inc., San Francisco, CA, USA, 47-59.

[3] Erica Cosijn and Peter Ingwersen. 2000. Dimensions of Relevance. Inf. Process. Manage. 36, 4 (July 2000), 533-550.

[4] K. Tamsin Maxwell and Burkhard Schafer. 2008. Concept and Context in Legal Information Retrieval. In Proceedings of the 2008 Conference on Legal Knowledge and Information Systems: FURIX 2008: The Twenty-First Annual Conference. IOS Press, NLD, 63-72.

[5] Alistair Moffat, Falk Scholer, Paul Thomas, and Peter Bailey. 2015. Pooled Evaluation Over Query Variations: Users Are as Diverse as Systems. In Proceedings of the 24th ACM International on Conference on Information and Knowledge Management (Melbourne, Australia) (CIKM '15). Association for Computing Machinery, New York, NY, USA, 1759-1762. https://doi.org/10.1145/2806416.2806606

[6] José Saias and Paulo Quaresma. 2002. Semantic enrichment of a web legal information retrieval system. In In Trevor Bench-Capon, Aspassia Daskalopulu, and 
Radboud Winkels, editors, Legal Knowledge and Information Systems. IOS. Press, 11-20.

[7] P. Samimi and S.D. Ravana. 2014. Creation of reliable relevance judgments in information retrieval systems evaluation experimentation through crowdsourcing. a review. Scientific World Journal. 14 (May 2014). https://doi.org/10.1155/2014/ 135641
[8] Kees van Noortwijk. 2017. Integrated legal information retrieval: new developments and educational challenges. European fournal of Law and Technology 8, 1 (2017). http://ejlt.org/article/view/544

[9] Marc Van Opijnen and Cristiana Santos. 2017. On the Concept of Relevance in Legal Information Retrieval. Artif. Intell. Law 25, 1 (March 2017), 65-87. https: //doi.org/10.1007/s10506-017-9195-8 